\title{
Efficacy of Transcutaneous Bilirubinometry as Compared to Serum Bilirubin in Preterm Newborn During Phototherapy
}

Sunil Gothwal ( $\nabla$ dr.sunilgothwal@gmail.com )

SMS Medical College and J K Lon Hospital

Neelam Singh

SMS Medical College Jaipur Rajasthan India

Sadasivam Sitaraman

SMS Medical College Jaipur Rajasthan India

Ramesh Choudhary

SMS Medical College Jaipur Rajasthan India

Kailash Kumar Meena

SMS Medical College Jaipur Rajasthan India

Ghan Shyam Bairwa

Rajasthan University of Health Sciences Jaipur

Mohan Bairwa

AllMS New Delhi India

Amrit Jeevan

NWRH Burnie TAS Australia

\section{Research Article}

Keywords: Preterm, Newborns, Transcutaneous, Bilirubin

Posted Date: April 12th, 2021

DOl: https://doi.org/10.21203/rs.3.rs-380978/v1

License: (1) (1) This work is licensed under a Creative Commons Attribution 4.0 International License. Read Full License 


\section{Abstract}

Transcutaneous measurement of bilirubin is being used for neonatal jaundice. Its utility during phototherapy in preterm babies is not established. Objective of our study was to assess the efficacy of transcutaneous bilirubin (TcB) measurement in comparison to total serum bilirubin in preterm newborns at admission and during phototherapy at covered skin area (glabella). It was a prospective observational study and conducted at neonatal intensive care unit of a tertiary care hospital from January 2017 to January 2019. One hundred eligible preterm neonates were enrolled. Babies who were very sick, with poor peripheral circulation, edematous, having conjugated hyperbilirubinemia, with major congenital malformations, already received phototherapy or exchange transfusion were excluded. Paired total serum bilirubin and transcutaneous bilirubin were measured at admission, at 6 hours and 24 hours during phototherapy. TcB was measured from area (glabella) covered by eye protector during phototherapy. Sample for TsB was taken within 10 minutes of TcB measurement. The mean difference between TsB and TCB values at admission, 6 hours and 24 hours of phototherapy were $-0.005(0.353),-0.350(0.611)$, and $-0.592(0.353)$ respectively. At admission or before starting of phototherapy the difference (TsB-TcB) was statistically not significant $(p=.125)$, while the difference in these values were statistically significant at 6 hours and 24 hours of phototherapy. Conclusion: TcB measurements from covered skin area in jaundiced preterm infants during phototherapy were not correlated with TsB and cannot be used as an alternate of serum bilirubin levels.

\section{What Is Known}

- Total serum bilirubin (TB) measurement in jaundiced neonates by high performance liquid chromatography is a "gold standard".

- There is evidence for use of transcutaneous bilirubinometry for assessment of bilirubin in term newborn.

\section{What is New}

- TCB measurements from a covered skin area in jaundiced preterm newborns under phototherapy were not correlated significantly at 6 hours and 24 hours of phototherapy, but correlated before phototherapy.

- TcB cannot be used as an alternate of serum bilirubin levels however, it reduces the frequency of blood sampling, iatrogenic blood loss, pain associated with prick and improves quality of neonatal care.

\section{Introduction}

Hyperbilirubinemia is a common occurrence in early neonatal period. About $50 \%$ of term and $80 \%$ of preterm neonates have serum bilirubin levels greater than $5 \mathrm{mg} / \mathrm{dl}$ in first few days of life. The levels may 
go up to $15 \mathrm{mg} / \mathrm{dl}$ in $6 \%$ of term neonates [1]. In preterm populations the levels are higher and have more serious implications. Timely phototherapy is the mainstay of treatment to avoid exchange transfusion.

Total serum bilirubin (TsB) is the gold standard to estimate serum bilirubin levels in jaundiced preterm neonates. It, however, requires a needle prick. Transcutaneous bilirubinometry (TcB), on the other hand, is quick, handy and non-invasive way of screening for hyperbilirubinemia requiring intervention. It has also been validated to assess jaundice in term neonates at the time of discharge [2]. Once commenced, phototherapy leads to bleaching of the skin, rendering further TcB measurements unreliable. Covering a part of the skin during phototherapy has been shown to give better correlation with TsB values in some studies [3-8]. However, there has been conflicting evidence in studies [9, 10]. These studies were heterogeneous in regards to gestational age and were done in small number of babies.

Hence, we planned to do an observational study to assess the accuracy of TcB levels on covered part of the skin, as compared to corresponding TsB levels in jaundiced preterm neonates before the start and during phototherapy.

\section{Trial design and participants:}

This single center, prospective observational study was conducted in the neonatal intensive care unit of a tertiary care hospital, in northern India from January 2017 to January 2019. Preterm neonates requiring phototherapy in first two weeks of life were considered eligible for enrolment into the study. Neonates with poor peripheral perfusion, sepsis, conjugated hyperbilirubinemia, having major congenital malformations, and hydrops fetalis were excluded. Those neonates who had already received phototherapy, ones who required exchange transfusion, were also excluded. We did not include babies with hemangioma or bruise on glabella that may have interfered with TcB measurements.

Case Enrollment: Preterm neonates with clinical neonatal jaundice were accessed for eligibility. After parental consent, the TcB /TsB levels were measured. As we enrolled Indian Blacks only, hence we did not take skin pigmentation into account.

Bilirubin measurement protocol: In all study participants, TcB levels were measured simultaneously with TsB. Serum bilirubin levels were measured at three defined time points, just before starting phototherapy, at 6 hours and 24 hours of continuous phototherapy. During the pilot study, we measured TcB at two different locations: on the covered skin area (glabella) under the eye protector and on the exposed naked skin close to it. We observed higher TcB values under the covered skin than surrounding uncovered skin area in the pilot study. For TsB measurement, 1-1.5 ml of blood was collected from each neonate. We used venous blood instead of capillary blood as the previous has lesser environmental effect and more accurate values than capillary blood as mentioned in previous study [11]. At our centre we were using venous blood more frequently than capillary blood for bilirubin measurement.

Peripheral venous blood was collected in plain vials, which was processed in the laboratory immediately. Our study centre was a dedicated pediatric hospital. The measurement of TsB was calibrated as per the 
reference range for neonate. Bilirubin assay was done by using diazo method. The principal of bilirubin assay was based on the Jendrassik-Grof method in which diazotized sulfanilic acid reacts with bilirubin to form azo-bilirubin, the latter of which was detected at an Optical Density of $540 \mathrm{~nm}$. Blood sampling was performed within 10 min of transcutaneous measurement of bilirubin.

Protocol for starting phototherapy: The decision to start phototherapy was based on clinical observation and serum bilirubin level by a pediatrician or a neonatologist. In our unit we followed NICE (National Institute for Health and Clinical Excellence) guidelines for phototherapy in preterm neonates set by National Collaborating Centre for Women's and Children's Health [12]. Preterm neonates with clinical jaundice were tested for TcB and TsB at admission. If the value of TcB/TsB was below the threshold for commencing phototherapy, the neonate was excluded from the study. Phototherapy was started if the value of $T c B / T s B$ was higher than the threshold value. In case of different values between the TcB and $T s B$, we took TsB value for the point of action. Exchange transfusion was done if values of serum bilirubin were higher than reference range or signs of acute bilirubin encephalopathy were present.

FANEM Bilitron Sky 2006, Super LED Phototherapy was used in our study. The distance between baby and phototherapy unit was $30 \mathrm{~cm}$. Irradiance was kept in the range of $30-50 \mathrm{micro} W / \mathrm{cm} 2 / \mathrm{nm}$ and measured the same by hand held radiometer. We used uniform phototherapy machine for all study participants and the light spectrum was maintained as per standard guidelines.

BiliCare device (BiliCare REF 81000300, Israel, 2015) was used to measure TcB. As per manufacturer's recommendation, the device was calibrated each time before use. It gave the average bilirubin value in $\mathrm{mg} / \mathrm{dl}$ of three back to back applications. During the treatment with phototherapy the neonates remained naked, with diaper and eye protector. As babies eyes were always covered with eye protector during phototherapy, we chose glabella, the area between the eyes, to measure TcB levels to correlate with TsB levels at same time points. We used neonatal eye protector of size $10 \mathrm{~cm} \times 3 \mathrm{~cm}$, opaque, non allergic and unique design manufactured by Ibis Medical, Kerala, India. It has a stretchable rim that fits in the head and minimized the chances of slip. In case of any accidental slip of the eye protector, the nursing staff on duty had placed it in same position.

Phototherapy unit was turned off only during TcB/TsB measurements and feeding. All the doctors involved in the care of these neonates were familiar with the use of BiliCare device as we have been using it for over two years in our nursery. We also performed three live demonstration of its use before the start of the study among our neonatal team.

\section{Sample size:}

Observations from study conducted by Lucanova et. al. [9] indicated that phototherapy significantly interferes with the accuracy of transcutaneous bilirubinometry. In order to have a correlation coefficient of 0.287 , a sample size of 94 neonates was estimated for a study power of $80 \%$ and two tailed alpha of 0.05. This was further rounded off to 100 neonates. 
Statistical analysis: Nonparametric tests were used for the outcomes on continuous scale. The mean of TcB for each measurement time point was compared with the corresponding TsB concentration. Pearson correlation coefficient ( $r$ ) analysis was performed to assess correlation coefficient between TcB and TsB. In order to assess the accuracy of noninvasive bilirubin concentration measurement, difference $(\Delta)$ between TsB and TcB and their 95\% confidence intervals ( $\mathrm{Cl} 95 \%)$ were evaluated. A P-value of 0.05 was considered statistically significant. The method of Bland and Altman was used to evaluate the agreement between bilirubin levels in blood and skin.

Ethical Approval: Written informed consent was taken from parents or caregiver of the baby before enrollment into the study. The study was approved by institutional Ethics Committee (letter no: 2823 $\mathrm{MC} / \mathrm{EC} / 2016)$.

\section{Results}

We had included neonates of resident Indian blacks in the population studied. Nine hundred newborns were admitted in the neonatal intensive care unit during the study period. Out of them 150 preterm newborns were diagnosed for neonatal jaundice and examined for eligibility. Fifty preterm neonates were excluded from the study due to poor peripheral perfusion $(n=5)$, sepsis $(n=15)$, already received phototherapy $(n=4)$, received exchange transfusion $(n=1)$, conjugated hyperbilirubinemia $(n=6)$, having major congenital malformations $(n=6)$, hydrops fetalis $(n=2)$, refusal for consent $(n=9)$ and other reasons $(n=2)$. One hundred newborns confirmed the eligibility and participated in the study. All enrolled cases $(n=100)$ were completed the study and results were analyzed.

The TcB and TsB at admission, 6 hours and 24 hours during phototherapy were measured for all study participants. Hence, total 300 paired samples of TcB and TsB were taken from 100 enrolled newborns. The Baseline characteristics were given in Table 1. Mean maternal age, birth weight and gestational age were 27.89 years, $1488.1 \mathrm{gms}$ and 37.76 hours respectively. Only $68 \%$ babies received full enteral feeding. Use of antenatal corticosteroids 52\% ( $n=52)$, mean Apgar@1 min was 7, mean Apgar@5 min was 9, 78 cases had CRIB-II score @ Level1, 22 cases had CRIB-II score @ Level 2 and no serious co morbidities were observed in the study subjects. The mean TCB (SD) levels at admission, 6 hours and 24 hours of phototherapy 14.7 (1.46), 13.58 (1.51) and 11.39 (1.42) $\mathrm{mg} / \mathrm{dl}$ while mean TsB (SD) levels at admission, 6 hours and 24 hours 14.08 (1.51), 13.93 (1.29) $11.98(1.32) \mathrm{mg} / \mathrm{dl}$.

The mean difference (SD) between TsB and TcB values at admission, 6 hours and 24 hours of phototherapy were $-0.005(0.353),-0.350(0.611)$, and $-0.592(0.353)$ respectively (Table 2$)$. At admission or before starting of phototherapy the difference (TsB-TcB) was statistically not significant $(p=.125)$, while the difference in these values were statistically significant at 6 hours and 24 hours of phototherapy.

The sensitivity and specificity for Tcb and TsB values at admission, 6 hours and 24 hours of phototherapy for serum bilirubin level $11,13,15 \mathrm{mg} / \mathrm{dl}$ was given in table 3 . Figures $1,2,3$ were showing Bland Altman plot for agreement between these two tests at above mentioned time points. 


\section{Discussion}

Phototherapy decreases bilirubin through photoisomerisation, structural isomerisation and photo oxidation [13]. Transcutaneous bilirubin estimation has proven efficacy for screening hyperbilirubinemia in full term neonates. A systematic review concluded that TcB devices reliably estimated bilirubin levels in preterm infants and also could be used to reduce blood sampling in preterm newborns [14]. A metaanalysis by Nagar et al resulted that TcB devices reliably estimate bilirubin level in preterm infants [15]. The evidence for its use in preterm neonates during phototherapy is lacking. We conducted this observational study in this selected group to assess correlation of TcB with serum bilirubin before commencement and during phototherapy. Our results showed statistically significant difference between TsB and TcB at 6 hours and 24 hours of phototherapy over covered skin area; although the difference was not statistically significant at starting of phototherapy.

Similar to other studies $[5,9]$ we used glabella as a site for transcutaneous measurement of bilirubin. Ozkan $\mathrm{H}$ et al and De Luca D et al covered the skin with a patch size of $2.5 \mathrm{~cm}$, while we covered with $10 \times 3 \mathrm{~cm}$ eye protector during the phototherapy $[7,25]$. Lucanova et al. measured transcutaneous bilirubin from abdomen [9]. Sajjadian, on the other hand, measured transcutaneous bilirubin from both forehead and sternum and in both healthy and sick newborns [16]. It was on priority for the clinicians to look for unexposed alternative sites for TcB measurement. As the genitals and eyes are covered in all babies receiving phototherapy, these alternative measurement sites may prove convenient for the same. Hence we used glabella for measurement of TcB in our study.

The phototherapy is used for treatment of neonatal hyperbilirubinemia to protect brain from the unconjugated bilirubin fraction. Transcutaneous bilirubinometry provides a rapid estimate of total serum bilirubin and can be helpful in monitoring the trends as it can be repeated, non invasive, reproducible, easier to perform and cost effective than TsB.

TcB cannot substitute TsB as the correlation between these two values is not well established. However, TcB does reduce the frequency of blood sampling and iatrogenic blood loss leading to anemia. It minimizes the pain and stress associated with prick. This noninvasive measurement is useful to improve quality of neonatal care. Bhutani et al found that frequent TcB and/or TsB measurement helps in monitoring of severe jaundice, thus reducing incidence of kernicterus [17].

Use of transcutaneous bilirubinometry is debatable in monitoring of newborns during phototherapy. There may be difference in $\mathrm{TCB}$ and $\mathrm{TsB}$ as bilirubin concentration in subcutaneous tissue exposed to phototherapy decreases before TsB concentration [18]. The exact time needed for the establishment of the equilibrium between the skin bilirubin to serum bilirubin is not known.

In our study, the correlation coefficient ( $r$ ) for difference in TsB and TcB were 0.024 at starting of phototherapy, 0.14 at 6 hours and 0.088 at 24 hours of phototherapy. These results were not correlated at 6 and 24 hours of phototherapy and had positive correlation at the starting of phototherapy. The correlation coefficient of present study was in contrast range of previous studies that ranged from 0.68 to 
0.96 [16, 17-19]. TsB was measured over sternum [20] and forehead, sternum, abdomen [21] while, we measured it over glabella. Recently, Alsaedi et al. [20] showed a significant correlation between TcB and TsB before the start and during phototherapy $(r .0 .85 ; p<0.001$ and $r .80 .0 ; p<0.001)$ in term neonates. The mean difference between TsB and TcB at the start was $0.36(1.36) \mathrm{mg} / \mathrm{dl}$, whereas it was -0.16 (1.37) $\mathrm{mg} / \mathrm{d} /$ during the treatment while we have $-0.05(.0353),-0.350(.0611)$ and $-0.592(.0353) \mathrm{mg} / \mathrm{dl}$ at admission, at 6 hours and at 24 hours of phototherapy. However, our study population was preterm neonates.

Cucuy et al [21] studied the correlation between TcB and TsB before, during, and after phototherapy in preterm babies. The overall correlation coefficient between TcB and TsB was 0.8. In contrast to our study they concluded that TcB is a reliable measure of jaundice before 35 weeks' gestation. Pendse et al. [22] used aluminum photoprotection system with $\mathrm{JM}-105$ and highlighted positive correlation in infants less than 32 weeks in contrast to our study.

Similar to our findings Lucanova et al [9], Murali et al [10] and Radfar et al [23] found that TcB was poorly correlated with TsB during phototherapy, irrespective of the site of measurements. Murali et al and Lucanova et al also reported a bad correlation between the TsB and TcB after the discontinuation of phototherapy $[9,10]$. However, we measured TcB and TsB upto 24 hours of phototherapy. Above all studies concluded that TCB does not appear to be reliable for estimating bilirubin in preterm. Luca D D et al obtained readings from the forehead and found a good correlation after $4-6 \mathrm{~h}$ from the start of phototherapy with a trend of increased difference between Tsb and Tcb with duration of phototherapy [24]. We used BiliCare device for measurement of TcB similar to other studies [10, 11], while Lucanova et al [9], used JM-103 similar to other studies $[8,11,19]$. Raimondi et al compared three most commonly used devices for TCB measurements i.e. BiliCare, JM103 and BiliMed. Pearson coefficients in their study showed good results for Bilicheck $(r=0.86)$ and JM-103 $(r=0.85)$ but poor for BiliMed $(r=0,70)$. Bilicheck and JM-103 had a greater area under the curve than BiliMed when TSB $=14 \mathrm{mg} / \mathrm{dl}$ was chosen as a threshold value. They concluded that, BiliCare and JM103 (but not BiliMed), are equally reliable [25].

Similar to Taylor JA et al study, we did not find any effect of birth weight, gestational age and other variables on correlation between TsB and TcB [26]. Although, most of the studies included only stable newborns. Jeon $\mathrm{J}$ et al studied the correlation in sick newborns and found significant correlation between TsB and TcB [27].

The strength of our study includes a prospective study with an adequate sample size. We restricted our study to stable preterm infants. We measured TcB and TsB at different time points during phototherapy. However, our study has certain limitations. Our all results were limited to Indian Black neonates. We obtained TcB upto 24 hours of continuous phototherapy instead of whole duration. Studies with longer point of time should address the correlation of TcB and TsB during the entire time of phototherapy. Likewise, we did not address the cost effectiveness of TcB as well.

The correlation of TsB and TcB before starting phototherapy was significant. But at 6 hours and at 24 hours of phototherapy the correlations were not significant. So TcB measurement should not be 
considered a surrogate for the gold standard TsB measurement during phototherapy. In these cases, TsB should be strongly considered.

\section{Conclusion}

Our study concluded that TCB measurements taken from a covered area of the skin in jaundiced, healthy, preterm newborns under phototherapy not correlated significantly at 6 hours and 24 hours of phototherapy, but correlated before phototherapy. Hence, TcB cannot be used as an alternate of serum bilirubin levels. However, TcB does reduce the frequency of blood sampling, iatrogenic blood loss, minimizes the pain associated with prick and useful to improve quality of neonatal care.

\section{List Of Abbreviations In Alphabetical Order}

Cl: Confidence Interval

Correlation coefficient ( $r$ )

LED: Light Emitting Diode

SD: Standard Deviation,

TcB: Transcutaneous Bilirubin,

TsB: Total Serum Bilirubin,

\section{Declarations}

\section{Author Declarations:}

Funding: There was no funding source

Conflicts of interest/Competing interests: We have no conflict of interest

Availability of data and material: NA

Code availability: NA

Authors' contributions: Study conception and design: SG, SS, RC, GSB, NS, Acquisition of data: Analysis and interpretation of data: SG, SS, RC, NS, AJ, MB, Drafting of manuscript: SG, RC, NS, AJ, GSB,MB, Critical revision: SG, SS, GSB, AJ,MLB, Final approval of manuscript: SG, SS, RC, GSB, AJ, NS,MB

Ethics approval: Ethical approval: Approved by Institutional Ethics Committee- $2823 \mathrm{MC} / \mathrm{EC} / 2016$ Consent to participate/ publication: Written informed consent was taken from parents/ guardian. 


\section{References}

1. Woodgate P, Jardine LA. Neonatal jaundice. BMJ Clin Evid. 2011;2011:0319

2. Mishra S, Chawla D, Agarwal R, Deorari AK, Paul VK (2010). Transcutaneous Bilirubin Levels in Healthy Term and Late Preterm Indian Neonates: Original Article. Indian J Pediatr 2010; 77 (1):45-50

3. C. A. Reyes, D. R. Stednitz, C. Hahn, K. D. Mutchie, S. R. McCullough, and K. Kronberg (2008), "Evaluation of the Bilichek being used on hyperbilirubinemic newborns undergoing home phototherapy," Archives of Pathology \& Laboratory Medicine 2008; 132 (4): 684-689,.

4. E. Zecca, G. Barone, D. De Luca, R. Marra, E. Tiberi, and C. Romagnoli (2009), "Skin bilirubin measurement during phototherapy in preterm and term newborn infants," Early Human Development 2009; 85(8) 537-540

5. R. Fonseca, R. Kyralessa, M. Malloy, J. Richardson, and S. K.Jain (2012), “Covered skin transcutaneous bilirubin estimation is comparable with serum bilirubin during and after phototherapy," Journal of Perinatology 2012; 32, (2), 129-131

6. Jangaard KA, Curtis H, Goldbloom RB (2006). Estimation of bilirubin using BiliChek, a transcutaneous bilirubin measurement device: effects of gestational age and use of phototherapy. Paediatr Child Health;11: 79-83.

7. Ozkan H, Oren H, Duman N, Duman M (2003). Dermal bilirubin kinetics during phototherapy in term neonates. ActaPaediatr; 92 (5): 577-581

8. Costa-Posada, U., Concheiro-Guisán, A., Táboas- Ledo, M.F. et al (2020). Accuracy of transcutaneous bilirubin on covered skin in preterm and term newborns receiving phototherapy using a JM-105 bilirubinometer. J Perinatol; 40: 226-231

9. Lucanova LC, Matasova K, Zibolen M, Krcho P (2016) .Original Article. Accuracy of transcutaneous bilirubin measurement in newborns after phototherapy Journal of Perinatology; 36 (10):858-61

10. Murli L, Thukral A, Sankar MJ, et al (2017). Reliability of transcutaneous bilirubinometry from shielded skin in neonates receiving phototherapy: a prospective cohort study. J Perinato/ 2017; 37 (2):182-187,

11. Mussavi M, Niknafs P, Bijari B. Determining the Correlation and Accuracy of Three Methods of Measuring Neonatal Bilirubin Concentration. Original Article.2013; Iranian Journal of Pediatrics;; 23 (3) :333-339

12. Neonatal Jaundice. Clinical Guideline May 2010 Funded to produce guidelines for the NHS by NICE, Published by the Royal College of Obstetricians and Gynaecologists, 27 Sussex Place, Regent's Park, London NW1 4RG; 1:1-495 .https://www.nice.org.uk/guidance/cg98/evidence/full-guideline245411821

13. Itoh S, Okada H, Kuboi T , Kusaka T (2017). Phototherapy for neonatal hyperbilirubinemia: Review

14. Article. Pediatrics International; 59: 959-966

15. Nagar G, Vandermeer B, Campbell S, Kumar M (2013). Reliability of transcutaneous bilirubin devices in preterm infants: a systematic review. Pediatrics; 132:871-81. 
16. Nagar G, Vandermeer B, Campbell S, Kumar M (2016). Effect of Phototherapy on the Reliability of Transcutaneous Bilirubin Devices in Term and Near-Term Infants: A Systematic Review and MetaAnalysis. Neonatology. 109(3):203-12.

17. Negar Sajjadian, Hamideh Shajari, Zeinab Saalehi, Fatemeh Esphahani, and Paymaneh Alizadeh Taheri (2012). Transcutaneous Bilirubin Measurement in Preterm Neonates. Original Article. Acta Medica Iranica; 50 (11): 765-70,

18. Bhutani, V., Vilms, R, Hamerman-Johnson, L (2010). Universal bilirubin screening for severe neonatal hyperbilirubinemia J Perinato/,30, S6-S15,

19. Juster-Reicher A, Flidel-Rimon O, Rozin I, Shinwell ES (2015). Correlation of transcutaneous bilirubinometry (TcB) and total serum bilirubin (TsB) levels after phototherapy. $J$ Matern Fetal Neonatal Med ; 28(11): 1329-1331

20. L Stillova, K Matasova, M Zibolen,et al (2009). Short Communication. Transcutaneous Bilirubinometry in Preterm Neonates. Indian Pediatrics ; 46, 405-408

21. Alsaedi SA (2018). Transcutaneous Bilirubin Measurements Can Be Used to Measure Bilirubin Levels during Phototherapy. Int J Pediatr, 4856390,

22. Cucuy M, Juster-Reicher A, Flidel O, Shinwell E (2018). Correlation between transcutaneous and serum bilirubin in preterm infants before, during, and after phototherapy. J Matern Fetal Neonatal Med. 31(10):1323-1326.

23. Pendse A, Jasani B, Nanavati R, Kabra N (2017). Comparison of transcutaneous bilirubin measurement with total serum bilirubin levels in preterm neonates receiving phototherapy. Indian Pediatr ; 54:641-3.

24. Radfar M, Hashemieh M, Shirvani F, Madani R (2016). Transcutaneous bilirubinometry in preterm and term newborn infants before and during photo-therapy. Arch Iran Med;19: 323-8.

25. De Luca D, Dell'Orto V (2017). Patched skin bilirubin assay to monitor neonates born extremely preterm undergoing phototherapy. J Pediatr; 188:122-7.

26. Raimondi F, Lama S, Landolfo F, et al (2012). Measuring transcutaneous bilirubin: a comparative analysis of three devices on a multiracial population. BMC Pediatr, $14 ; 12: 70$

27. Taylor JA, Burgos AE, Flaherman V et al. Better Outcomes through Research for Newborns Network. Discrepancies between transcutaneous and serum bilirubin measurements. Pediatrics. 2015 Feb; 135 (2):224-31.

28. Jeon J, Lim G, Oh KW, Lee NM, Park HW, Chung ML. The forehead is a better site than the sternum to check transcutaneous bilirubin during phototherapy in sick infants. BMC Pediatr. $2020 \mathrm{DeC}$ 5;20(1):548.

\section{Tables}

Table 1: Baseline characteristics of the study participants 


\begin{tabular}{|c|c|c|c|}
\hline Baseline Characteristics & Number & SD & $95 \% \mathrm{Cl}$ \\
\hline Male (\%) & $\begin{array}{l}50 \\
(50 \%)\end{array}$ & & \\
\hline Number of paired sample & 300 & & \\
\hline Mean maternal age (years) & 27.89 & 5.82 & $5.11-6.77$ \\
\hline Mean weight at birth ( gms) & 1488.1 & 264.37 & $\begin{array}{l}232.11 \text { - } \\
307.11\end{array}$ \\
\hline Mean gestational age & 32.76 & 1.82 & $1.60-2.12$ \\
\hline Mean postnatal age at the time of admission (hrs) & 72.36 & 264.37 & $16.46-21.77$ \\
\hline Number of neonates received enteral nutrition (\%) & $\begin{array}{l}68 \\
(68 \%)\end{array}$ & & \\
\hline Number of mothers received antenatal steroids (\%) & $\begin{array}{l}52 \\
(52 \%)\end{array}$ & & \\
\hline Mean Apgar Score @1 min & 7 & & \\
\hline Mean Apgar Score @5 min & 9 & & \\
\hline Number of neonates with CRIB-II score @ Level1 (\%) & $\begin{array}{l}78 \\
(78 \%)\end{array}$ & & \\
\hline Number of neonates with CRIB-II score @ Level 2 (\%) & $\begin{array}{l}22 \\
(22 \%)\end{array}$ & & \\
\hline Number of neonates with serious co morbidities (\%) & $0(0 \%)$ & & \\
\hline $\begin{array}{l}\text { Number of neonates required mechanical support/ } \\
\text { vasopressor (\%) }\end{array}$ & $0(0 \%)$ & & \\
\hline Mean weight loss in neonates since birth to admission (\%) & $4.4 \%$ & & \\
\hline
\end{tabular}

SD: Standard Deviation, Cl: Confidence Interval, CRIB: Clinical Risk Index For Babies

\section{Table 2: TcB and TsB measurements and correlation}




\begin{tabular}{|llllll|}
\hline $\begin{array}{l}\text { Difference b/w TcB and } \\
\text { TsB } \\
(\mathrm{n}=100)\end{array}$ & Mean (SD) & $\begin{array}{l}\text { Std. Error } \\
\text { Mean }\end{array}$ & $95 \% \mathrm{Cl}$ & $\mathbf{r}$ & Significance \\
\hline At admission & $\begin{array}{l}-0.005 \\
(0.3532)\end{array}$ & 0.0353 & $\begin{array}{l}-0.075 \text { to } \\
-0.065\end{array}$ & 0.154 & 0.125 \\
\hline At 6 hrs of phototherapy & $\begin{array}{l}-0.350 \\
(0.6108)\end{array}$ & 0.0611 & $\begin{array}{l}-0.471 \text { to } \\
-0.229\end{array}$ & 0.437 & 0.000 \\
\hline At 24hrs of phototherapy & $\begin{array}{l}-0.592 \\
(0.3532)\end{array}$ & 0.0353 & $\begin{array}{l}-0.662 \text { to- } \\
0.522\end{array}$ & 0.296 & 0.003 \\
\hline
\end{tabular}

TcB: Transcutaneous Bilirubin, TsB: Total Serum Bilirubin, SD: Standard Deviation, Cl: Confidence Interval

Table 3: - Sensibility and specificity of TcB to predict TsB at certain time points

\begin{tabular}{|lllll|}
\hline $\begin{array}{l}\text { Serum Bilirubin level }(\mathrm{mg} / \mathrm{dl}) \\
\text { Cut off value }\end{array}$ & & $\begin{array}{l}\text { At } \\
\text { Admission }\end{array}$ & $\begin{array}{l}\text { At 6 hours of } \\
\text { phototherapy }\end{array}$ & $\begin{array}{l}\text { At 24 hours of } \\
\text { phototherapy }\end{array}$ \\
\hline$>11 \mathrm{mg} / \mathrm{dl}$ & Sensitivity & $100 \%$ & $96.9 \%$ & $85.9 \%$ \\
\hline$>13 \mathrm{mg} / \mathrm{dl}$ & Specificity & - & $100 \%$ & $100 \%$ \\
\hline$>15 \mathrm{mg} / \mathrm{dl}$ & Sensitivity & $95 \%$ & $75.9 \%$ & $66.7 \%$ \\
\hline & Specificity & $90 \%$ & $100 \%$ & $100 \%$ \\
\hline & Sensitivity & 88.23 & 98.63 & - \\
\hline & Specificity & 86.36 & 100 & $100 \%$ \\
\hline
\end{tabular}

TcB: Transcutaneous Bilirubin, TsB: Total Serum Bilirubin

\section{Figures}




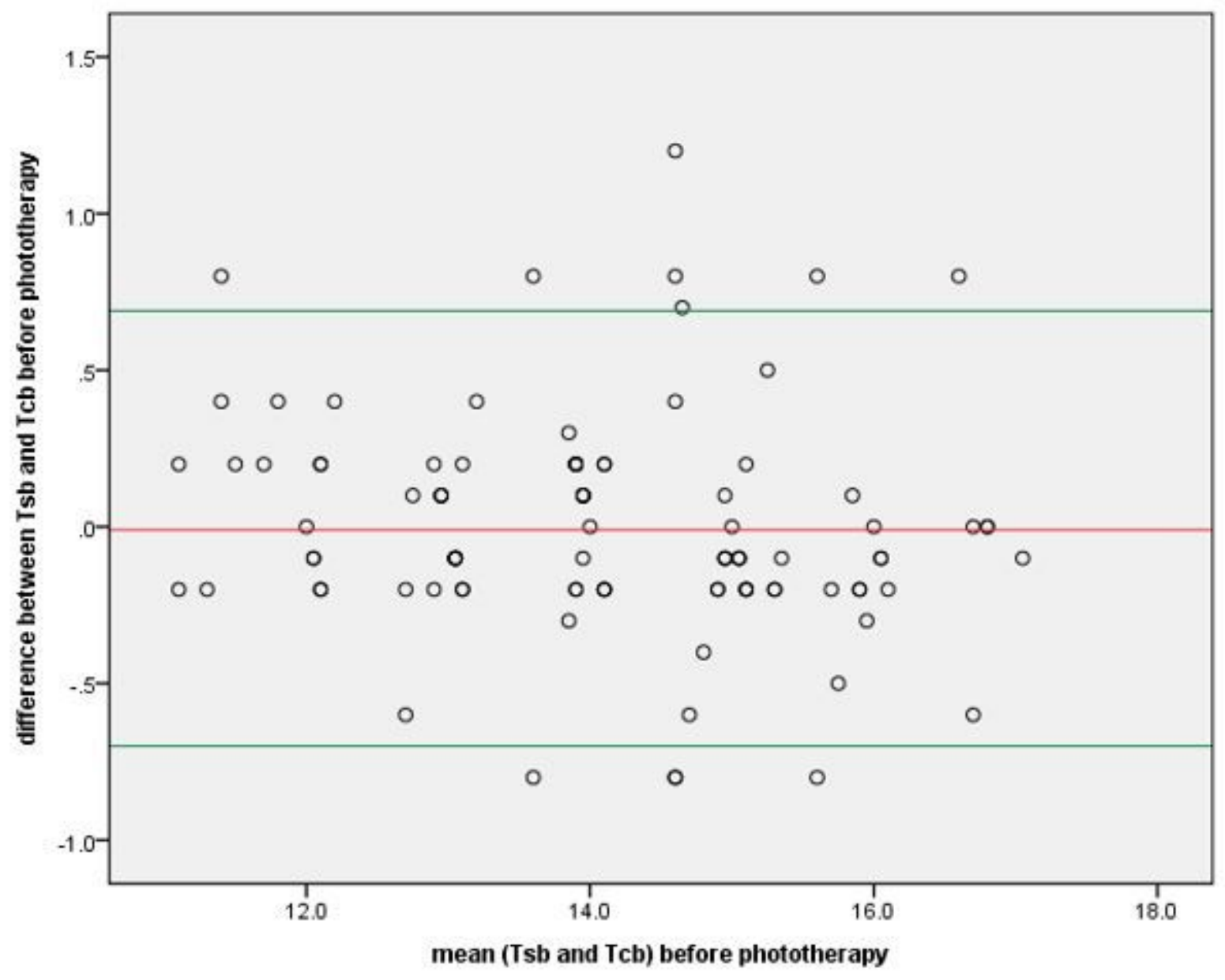

Figure 1

Bland Altman plot at admission 


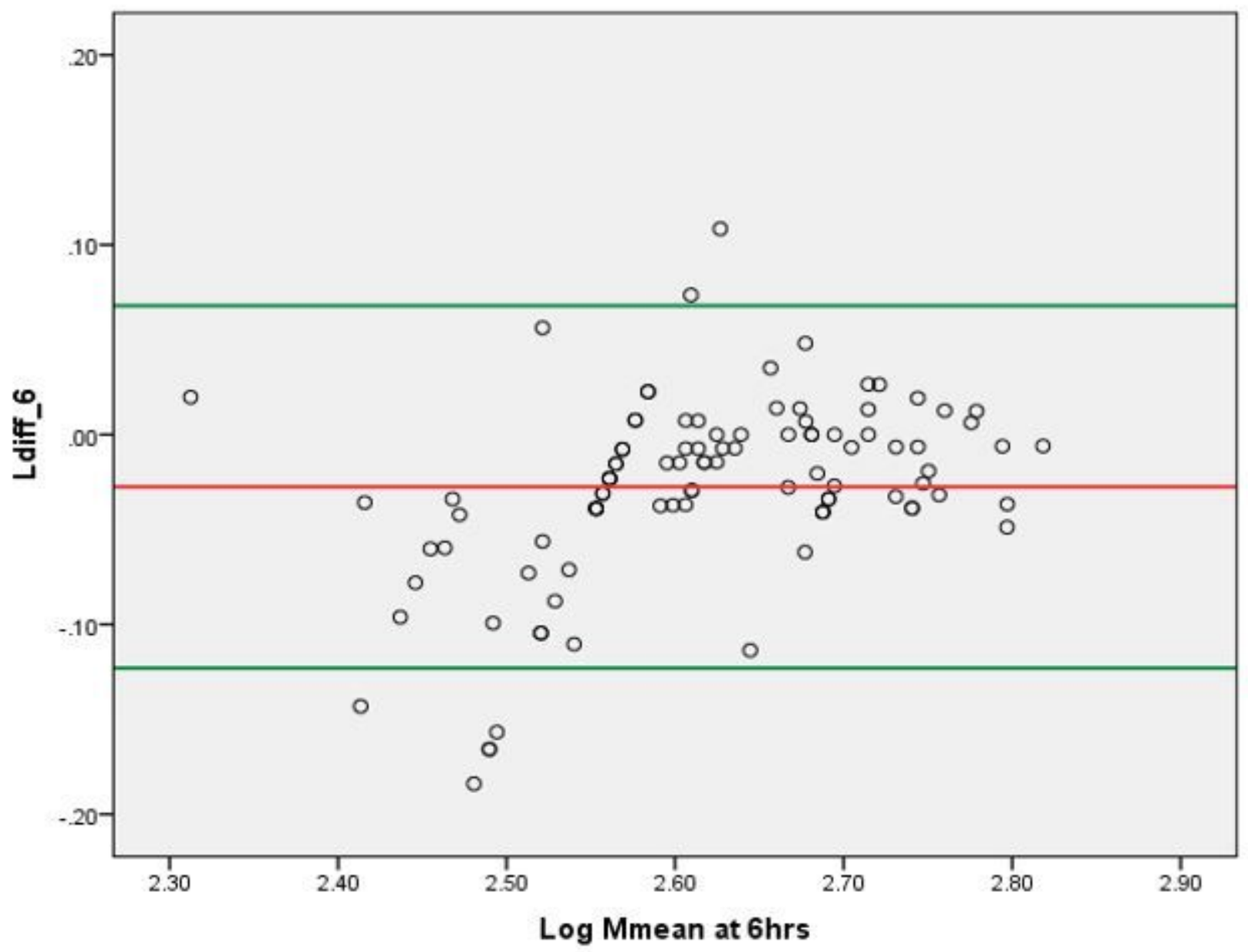

Figure 2

Bland Altman plot at 6 hours of Phototherapy 


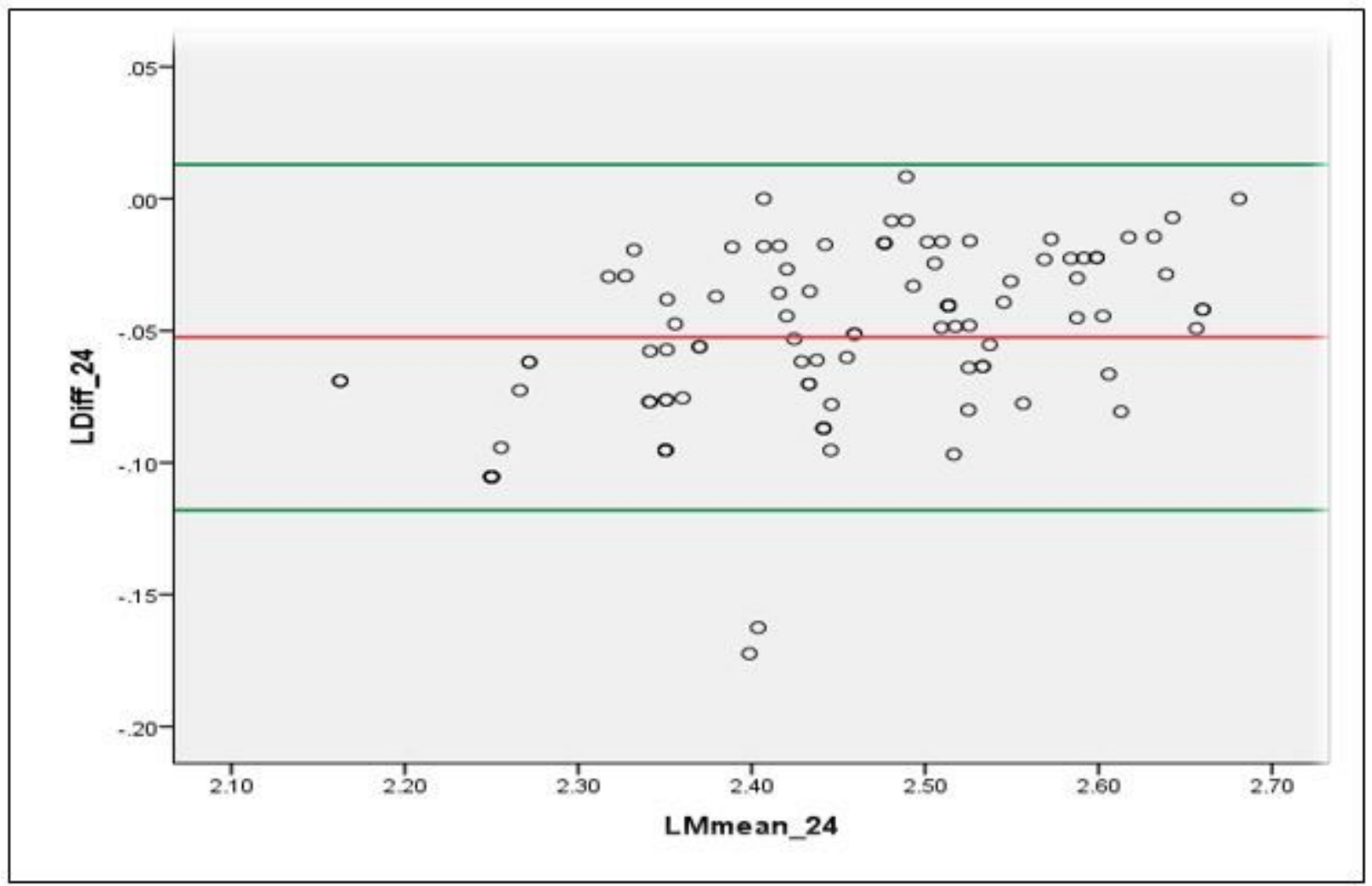

Figure 3

Bland Altman plot at 24 hours of Phototherapy 